\title{
Pulmonary Dysfunction in Patients With Femoral Shaft Fracture Treated WITH INTRAMEDULLARY NAILING
}

\author{
By Brent L. Norris, MD, W. Christopher Patton, MD, Joseph N. RudD JR., BSN, PhD, \\ ColleEn M. Schmitt, MD, MHS, AND JeFFrey A. Kline, MD \\ Investigation performed at the University of Tennessee College of Medicine, Chattanooga, Tennessee, \\ and the Carolinas Medical Center, Charlotte, North Carolina
}

\begin{abstract}
Background: This study was undertaken to determine whether alveolar dead space increases during intramedullary nailing of femoral shaft fractures and whether alveolar dead space predicts postoperative pulmonary dysfunction in patients undergoing intramedullary nailing of a femoral shaft fracture.

Methods: All patients with a femoral shaft fracture were prospectively enrolled in the study unless there was evidence of acute myocardial infarction, shock, or heart failure. Arterial blood gases were measured at three consecutive time-periods after induction of general anesthesia: before intramedullary nailing and ten and thirty minutes after intramedullary nailing. The end-tidal carbon-dioxide level, minute ventilation, positive end-expiratory pressure, and percent of inspired and expired inhalation agent were recorded simultaneously with the blood-gas measurement. Postoperatively, all subjects were monitored for evidence of pulmonary dysfunction, defined as the need for mechanical ventilation or supplemental oxygen (at a fraction of inspired oxygen of $>40 \%$ ) in the presence of clinical signs of a respiratory rate of $>20$ breaths/min or the use of accessory muscles of respiration.

Results: Seventy-four patients with a total of eighty femoral shaft fractures completed the study. Fifty fractures $(62.5 \%)$ underwent nailing after reaming, and thirty fractures (37.5\%) underwent nailing with minimal or no reaming. The mean alveolar dead-space measurements before canal opening and at ten and thirty minutes after canal opening were $14.5 \%, 15.8 \%$, and $15.2 \%$ in the total series of seventy-four patients (general linear model, $p=0.2$ ) and $20.5 \%, 22.7 \%$, and $24.2 \%$ in the twenty patients with postoperative pulmonary dysfunction (general linear model, $p=0.05$ ). Of the twenty-one patients with an alveolar dead-space measurement of $>20 \%$ thirty minutes after nailing, sixteen had postoperative pulmonary dysfunction. According to univariate and multivariate analysis, the alveolar dead-space measurement was strongly associated with postoperative pulmonary dysfunction.

Conclusions: According to our data, intramedullary nailing of femoral shaft fractures did not significantly increase alveolar dead space, and the amount of alveolar dead space can predict which patients will have pulmonary dysfunction postoperatively.
\end{abstract}

$\mathrm{L}$ ong-bone fractures, especially femoral shaft fractures, can contribute to lung damage in patients who have sustained a traumatic injury ${ }^{1-5}$. Fractures disrupt both the medullary vessels and the marrow matrix, creating the potential for extrusion of marrow contents into the venous system $^{4-6}$. A large bolus of embolized marrow can block perfusion to a portion of the well-ventilated lung, resulting in a high ventilation-to-perfusion ratio or increased alveolar dead space $^{7-17}$.

The method used to prepare the medullary canal may determine the amount of bone marrow extrusion during intramedullary nailing ${ }^{3,18-20}$. Pape et al. proposed that reaming of the femoral canal may exacerbate lung damage in patients with chest injury ${ }^{2-4}$. Reaming of the medullary canal may increase the risk of fat emboli and the prevalence of adult res- piratory distress syndrome in patients with concurrent pulmonary injury. Although the findings of Pape et al. ${ }^{2-4,20}$ have not been corroborated by other investigators ${ }^{21}$, concern remains regarding the effect of intramedullary nailing of femoral shaft fractures on the pulmonary function of patients with multiple injuries. If objective physiologic or clinical measures of pulmonary dysfunction were to show an adverse effect of nailing or reaming, then a compelling argument could be made against performing one or both procedures.

Previous investigators have used intraoperative echocardiography to demonstrate echogenic material passing through the right ventricle during femoral nailing; however, the findings were not correlated with pulmonary function ${ }^{6}$. We are aware of only one recent study in which pulmonary physiologic consequences of femoral nailing and/or intramedullary reaming were 
The Journal of BOnE \& JOINT SURGERY • JBIS. ORG Volume 83-A · Number 8 - August 2001
Pulmonary Dysfunction in Patients with Femoral Shaft Fracture Treated With Intramedullary NaIling documented in humans ${ }^{22}$. In that study, the alveolar dead space and alveolar-arterial gradient were evaluated before, during, and after nailing. However, few patients had an adverse pulmonary outcome, limiting the conclusions of the study. Measurement of alveolar dead space provides a clinical measure of pulmonary vascular occlusion, regardless of the etiology, because it accurately reflects the difference between measure-

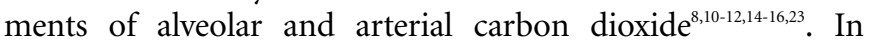
healthy patients, the ratio should approach 1.0 , as carbon dioxide rapidly debonds from the hemoglobin molecule at the alveolar level. Few clinical conditions affect the ratio acutely; pulmonary embolic events and extremely low cardiac flow states (shock) are the most common conditions that do affect the ratio. Therefore, measurement of this ratio should help to identify variables that affect the pulmonary system in patients with a femoral shaft fracture. Several questions may be addressed and hopefully answered with evaluation of the alveolar dead space: Do patients with a femoral shaft fracture have increased alveolar dead space as a result of the fracture? Does femoral nailing increase alveolar dead space? Does intramedullary reaming affect alveolar dead space? Moreover, a large alveolar dead space (as estimated by a high $\mathrm{Vd} / \mathrm{Vt}$ [ratio of physiologic dead space to tidal volume]) measured intraoperatively may influence postoperative management. The hypotheses of the present study were (1) the amount of alveolar dead space changes with intramedullary nailing of femoral shaft fractures, (2) reaming alters the amount of alveolar dead space, (3) measurement of alveolar dead space can predict postoperative pulmonary dysfunction, and (4) other variables such as the Injury Severity Score, pulmonary injury, and pulmonary comorbidities may more accurately predict pulmonary dysfunction following intramedullary nailing of femoral shaft fractures.

\section{Materials and Methods Study Enrollment}

This study was conducted at the University of Tennessee College of Medicine in Chattanooga, Tennessee, from January 1997 to January 1998, and at the Carolinas Medical Center in Charlotte, North Carolina, from February 1996 to June 1996. The study was approved by the institutional review boards of both hospitals. To be included in the study, a patient had to be an adult with a femoral shaft fracture that had occurred $\geq 5 \mathrm{~cm}$ distal to the level of the lesser trochanter and $\geq 5 \mathrm{~cm}$ proximal to the knee joint and was amenable to treatment with an intramedullary nail. Exclusion criteria included acute myocardial infarction, circulatory shock, and heart failure. An acute myocardial infarction was defined as an active injury current on electrocardiogram or biochemical evidence of acute cardiac muscle injury. Circulatory shock was defined as systolic blood pressure of $<90 \mathrm{~mm} \mathrm{Hg}$ together with base deficits (a measure of anaerobic metabolism secondary to poor tissue perfusion) of less than $-5 \mathrm{mN}$. Heart failure was defined as the presence of pulmonary rales on physical examination together with the appearance of alveolar edema on a chest radiograph as well as a cardiac index (if available) of $<2.5 \mathrm{~L} / \mathrm{min} / \mathrm{m}^{2}$. Initially, six patients were excluded from the study because of shock; however, these patients were later enrolled after the hemodynamic instability had been corrected. No patient was excluded because of myocardial infarction or heart failure.

\section{Collection of Preoperative, Intraoperative, and Postoperative Data}

Data for each patient were prospectively logged on a standardized form that included demographic information, time to fixation of the femoral fracture, associated injuries, Injury Severity Score, pulmonary injury (defined as a pneumothorax, hemothorax, or pulmonary contusion), pulmonary comorbidity (defined as asthma or chronic obstructive pulmonary disease), and smoking status. Additional data included intraoperative surgical technique (nail insertion technique and number of canal passes) and parameters associated with mechanical ventilation (fraction of inspired oxygen, minute ventilation, positive end-expiratory pressure, and percent of inspired and expired inhalation agent). We recorded the presence or absence of postoperative pulmonary dysfunction, which was defined as the need for mechanical ventilation or clinical signs of respiratory distress (the need for supplemental oxygen administration at a fraction of inspired oxygen of $>40 \%$ as well as a respiratory rate of $>20$ breaths/min or the use of accessory muscles of respiration). Accessory muscle use was defined as visible retraction of the sternocleidomastoid and intercostal muscles on physical examination. The fraction of inspired oxygen was calculated with the assumption of a 0.03 increase in the fraction of inspired oxygen per liter of supplemental oxygen per minute in a nonintubated patient. The specific guidelines for the use of mechanical ventilation were determined by the attending physicians but, in general, included clinical evidence of inadequate gas exchange and respiratory distress.

All subjects were evaluated during the first twenty-fourhour postoperative period for development of pulmonary dysfunction. Additional evaluations were performed at the request of the trauma team or the nurse treating the patient. All pulmonary evaluations and measurements were performed by either the authors or the orthopaedic trauma service without knowledge of the intraoperative pulmonary gas-exchange data. Arterial blood-gas levels, chest radiographs, and other clinically important data were obtained when the patient's condition warranted it. Other important outcomes that we recorded included readmittance to an intensive-care unit and death during hospitalization.

\section{Pulmonary Gas-Exchange Indexes}

Arterial blood-gas measurements were performed at three time-points: (1) after induction of anesthesia but before the beginning of the procedure, (2) ten minutes after the opening of the femoral canal, and (3) thirty minutes after the opening of the femoral canal. At the time of the arterial blood-gas measurement, end-tidal carbon-dioxide concentration, positive end-expiratory pressure, minute ventilation, and percent of inspired and expired volatiles (halothane, fluorane, and isoflurane) were also recorded. Anesthesia personnel who had been 
The Journal of BOne \& JOINT SURGERY • JBIS. ORG VOlume 83-A · Number 8 - August 2001
Pulmonary Dysfunction in Patients With Femoral Shaft Fracture Treated With Intramedullary Nailing instructed about the ongoing study performed the intraoperative pulmonary measurements. Capnometers (model 1025B; Hewlett-Packard, Atlanta, Georgia) were calibrated and maintained by hospital bioengineering personnel. The alveolar dead space was calculated with use of the Enghoff modification of the Bohr equation: $\mathrm{Vd} / \mathrm{Vt}=\left(\mathrm{PaCO}_{2}-\mathrm{PetCO}_{2}\right) /$ $\left(\mathrm{PaCO}_{2}\right) \times 100 \%$, where $\mathrm{PaCO}_{2}$ is the arterial partial pressure of carbon dioxide (obtained from the arterial blood-gas measurement) and $\mathrm{PetCO}_{2}$ is the steady-state end-tidal carbondioxide concentration measured by capnometry at each of the three time-points ${ }^{24}$.

\section{Operative Technique}

Patients were placed under general anesthesia with endotracheal intubation and with use of volume-controlled ventilation prior to any manipulation of the fracture. They were then positioned either supine on a fracture-table or supine with a hip-bump and the leg free on a radiolucent table. Associated injuries and the preference of the attending orthopaedic surgeon determined the positioning of the patient. All surgery was performed by orthopaedic surgery residents with the supervision of attending faculty. The femoral shaft fractures were fixed with the Synthes intramedullary nail (Paoli, Pennsylvania). A standard lateral hip incision was made proximal to the level of the greater trochanter, and a c-arm was used to visualize the proximal femoral starting point on both the anteroposterior and the lateral image. A 2.8-mm guide-pin was drilled through the piriformis fossa and into the medullary canal. The proximal part of the femur was then opened with a 13-mm cannulated reamer, and a femoral nail was placed with extensive reaming or with minimal or no reaming. The decision whether to ream was left to the attending surgeon. A single canal pass was recorded whenever an object (a guide-rod, awl, reamer, or nail) was passed into the opened canal, distal to the level of the isthmus, and then removed. Locking of the nail was performed on the basis of the fracture configuration and the preference of the attending surgeon. Patients were monitored postoperatively by the orthopaedic trauma service, and all complications were recorded. Postoperative mobilization and rehabilitation of the patients were advanced on the basis of the fracture configuration and the associated injuries.

\section{Data Analysis}

Data were entered on an Excel spreadsheet (version 4.0; Microsoft, Redmond, Washington, 1992). Analyses were performed with use of SPSS software (version 8.0.0; SPSS, Chicago, Illinois, 1997). Means, ranges, standard deviations, and frequency distributions for continuous variables were examined; proportions were used to describe categorical variables. The continuous variables were normally distributed; therefore, parametric methods were used for our analyses.

The primary study hypothesis (that the physiologic measure of alveolar dead space changes during nailing) was tested by evaluating alveolar dead space over time with repeated measures of analysis of variance. The second study hypothesis (that reaming of the femoral canal is associated with a change in alveolar dead space) was tested by examining the association between the extent of reaming and the change in alveolar dead space over time with use of a general linear model. The third study hypothesis (that increased alveolar dead space prior to or after surgical fixation is associated with postoperative pulmonary dysfunction) was tested with logistic regression analysis, with postoperative pulmonary dysfunction treated as the dependent variable. The following variables were examined for possible association with postoperative pulmonary dysfunction: alveolar dead space measured preoperatively and ten and thirty minutes after opening of the femoral canal, reaming, the Injury Severity Score, pulmonary comorbidity, smoking status, and age. Correlation between variables was examined with use of the Pearson method. Because odds ratios and confidence intervals are a clinically meaningful way to present these data, we converted beta coefficients (and standard error of beta) to respective odds ratios with $95 \%$ confidence intervals, in order to describe the association between predictors of postoperative pulmonary dysfunction and outcome. Finally, to test the fourth hypothesis, we used multivariate logistic regression analysis to evaluate the potential importance of other covariates, such as Injury Severity Score and the presence of pulmonary injury or comorbidity, by testing the association between these covariates and postoperative pulmonary dysfunction, with postoperative pulmonary dysfunction again treated as the dependent variable. Odds ratios and $95 \%$ confidence intervals were generated for the results of these multivariate models. We also examined the discriminate ability of our final model for predicting postoperative pulmonary dysfunction.

\section{Results}

\section{Clinical Data}

Seventy-four patients with a total of eighty femoral shaft fractures enrolled in and completed the study. The mean age of the patients was thirty-two years (range, sixteen to seventyseven years). Six patients (8\%) had a preinjury history of pulmonary comorbidity. Nineteen patients $(26 \%)$ had a history of pulmonary injury. Eleven of them had a pulmonary contusion (as diagnosed by chest radiography), and eight had a hemothorax, a pneumothorax, or a hemopneumothorax; all eight patients required placement of a chest tube. Twenty-five patients $(34 \%)$ reported a history of smoking. Thirty-nine patients $(53 \%)$ had multiple injuries, and thirty-five $(47 \%)$ had an isolated fracture. Seventy-four fractures in sixty-eight patients $(92 \%)$ were treated with intramedullary nailing less than twelve hours after the injury. Three fractures in three patients (4\%) initially were treated with external fixation, which was later converted to intramedullary nailing. Three patients with three fractures were managed with distal traction and then underwent nailing more than twelve hours after the injury. The mean Injury Severity Score was 17 points, with a range of 9 to 43 points. We compared baseline risk factors for postoperative pulmonary dysfunction between the patients who had extensive reaming and those who had minimal or no reaming. The patients with extensive reaming were more likely 
The JOURNAL OF BONE \& JOINT SURGERY · JBJS.ORG Volume 83-A · Number 8 - August 2001
Pulmonary Dysfunction in Patients with Femoral Shaft Fracture Treated With Intramedullary NaIling

TABLE I Alveolar Dead Space (Vd/Vt) for Various Subsets Within the Study Group

\begin{tabular}{|c|c|c|c|c|}
\hline & $\begin{array}{c}\text { Before } \\
\text { Canal Opening (\%) }\end{array}$ & $\begin{array}{c}10 \text { Min After } \\
\text { Canal Opening (\%) }\end{array}$ & $\begin{array}{c}30 \text { Min After } \\
\text { Canal Opening (\%) }\end{array}$ & $P$ Value \\
\hline All patients $(n=74)$ & $14.5 \pm 8.6$ & $15.8 \pm 9.6$ & $15.2 \pm 9.5$ & $0.2 *$ \\
\hline $\begin{array}{l}\text { No postop. pulmonary } \\
\text { dysfunction }(n=54)\end{array}$ & $12.2 \pm 7.5$ & $13.2 \pm 8.5$ & $11.8 \pm 7.5$ & $0.1 \dagger$ \\
\hline $\begin{array}{l}\text { Postop. pulmonary } \\
\text { dysfunction }(n=20)\end{array}$ & $20.5 \pm 8.5$ & $22.7 \pm 9.1$ & $24.2 \pm 8.2$ & $0.05 \dagger$ \\
\hline Extensive reaming $(n=50)$ & $14.8 \pm 8.7$ & $16.1 \pm 10.5$ & $13.9 \pm 10.6$ & \\
\hline Minimal or no reaming $(n=30)$ & $13.5 \pm 8.7$ & $15.0 \pm 8.3$ & $17.2 \pm 7.3$ & $0.03 \dagger$ \\
\hline
\end{tabular}

to have a history of smoking than were the patients with minimal or no reaming ( $\mathrm{p}=0.01$, chi square), but the two groups were similar with regard to the presence of pulmonary injury $(\mathrm{p}>0.05$, chi square) and the Injury Severity Score $(\mathrm{p}>0.05)$.

\section{Intraoperative Data}

No patient was excluded from the study because of preoperative or postoperative hemodynamic instability. The mean minute ventilation for all subjects was $7.8 \mathrm{~L}$. We observed no significant association between the alveolar dead-space measurements and minute ventilation, positive end-expiratory pressure, or percent inspired and expired volatiles (anesthetic agents). Fifty fractures $(62.5 \%)$ were treated with extensive reaming and the placement of a $12-\mathrm{mm}$-diameter femoral nail, and thirty $(37.5 \%)$ were treated with minimal or no reaming and the placement of a 9 or $10-\mathrm{mm}$-diameter femoral nail. The mean number of canal passes for these two subgroups was seven (range, five to eleven) and three (two, three, or four), respectively.

\section{Pulmonary Gas-Exchange Indexes}

Table I summarizes the alveolar dead-space (Vd/Vt) data. The mean alveolar dead-space measurements before canal opening, ten minutes after canal opening, and thirty minutes after canal opening were $14.5 \%, 15.8 \%$, and $15.2 \%$, respectively (general linear model, $\mathrm{p}=0.2$ ), for the seventy-four patients. No significant change in the alveolar dead space occurred between measurements. Thus, our data do not support the first hypothesis that intramedullary nailing of femoral shaft fractures alters alveolar dead space.

The mean alveolar dead-space measurements before canal opening, ten minutes after canal opening, and thirty minutes after canal opening were $14.8 \%, 16.1 \%$, and $13.9 \%$ for the patients who had nailing with extensive reaming and $13.5 \%$, $15.0 \%$, and $17.2 \%$ for those who had nailing with minimal or no reaming. The increase in the mean alveolar dead space over time among the patients who had minimal or no reaming was significantly greater than the increase in those with extensive reaming $(\mathrm{p}=0.03)$. Therefore, our data do not support the second hypothesis that extensive intramedullary reaming of femoral shaft fractures increases alveolar dead space.

\section{Postoperative Data}

According to our criteria, postoperative pulmonary dysfunction developed in twenty patients $(27 \%)$. Fifteen of these patients required mechanical ventilation postoperatively. Three of them were reintubated after attempted extubation following surgery. Other reasons for the mechanical ventilation included severe head injury (three patients), poor pulmonary mechanics (four patients), facial fractures (two patients), failure to extubate as ordered (one patient), and impending operative procedures by specialists in other fields of surgery (two patients). The mean alveolar dead-space measurements before canal opening, ten minutes after canal opening, and thirty minutes after canal opening were $20.5 \%, 22.7 \%$, and $24.2 \%$, respectively, in patients with postoperative pulmonary dysfunction. These values were significantly greater $(p=0.05)$ than those for the patients without postoperative pulmonary dysfunction. As described above, eight patients remained intubated for reasons possibly unrelated to pulmonary dysfunction. Six of these patients had been treated with extensive reaming while two had been treated with minimal reaming. When these patients were excluded from the analysis, the remaining patients with postoperative pulmonary dysfunction had no significant change in the mean alveolar dead space over time, although a trend was noted $(\mathrm{p}<0.08)$. This finding suggests that patients in whom pulmonary dysfunction develops after operative treatment of a femoral shaft fracture have an increased alveolar dead space prior to that treatment, supporting our third hypothesis.

In twenty-one patients, the alveolar dead-space measurement at thirty minutes was $>20 \%$. Sixteen $(76 \%)$ of these patients had evidence of postoperative pulmonary dysfunction, and one patient with a measurement of 52\% died during the study period. Postmortem examination confirmed fat emboli in the lungs. 
The Journal of Bone \& JOINT SuRgery • JBJS.ORG Volume 83-A · Number 8 - August 2001
Pulmonary Dysfunction in Patients with Femoral Shaft Fracture Treated With Intramedullary Nailing

\begin{tabular}{|c|c|}
\hline Variable & $\begin{array}{c}\text { Odds Ratio } \\
\text { (95\% Confidence } \\
\text { Interval)* }\end{array}$ \\
\hline \multicolumn{2}{|l|}{ Univariate model } \\
\hline $\mathrm{Vd} / \mathrm{Vt}$ before canal opening $\dagger$ & $1.14(1.05-1.24)$ \\
\hline $\mathrm{Vd} / \mathrm{Vt} 10$ min after canal opening $\dagger$ & $1.16(1.06-1.27)$ \\
\hline $\mathrm{Vd} / \mathrm{Vt} 30$ min after canal opening $\dagger$ & $1.43(1.19-1.72)$ \\
\hline Injury Severity Score $†$ & $1.10(1.03-1.19)$ \\
\hline Pulmonary injury/comorbidity $†$ & $4.14(1.15-14.9)$ \\
\hline Smoker & NS \\
\hline Age & NS \\
\hline \multicolumn{2}{|l|}{ Multivariate model } \\
\hline $\mathrm{Vd} / \mathrm{Vt}$ before canal opening & NS \\
\hline $\mathrm{Vd} / \mathrm{Vt} 10 \mathrm{~min}$ after canal opening & NS \\
\hline $\mathrm{Vd} / \mathrm{Vt} 30 \mathrm{~min}$ after canal opening $\dagger$ & $1.18(1.05-1.33)$ \\
\hline Injury Severity Score $\dagger$ & $1.11(1.01-1.21)$ \\
\hline Pulmonary injury/comorbidity & NS \\
\hline Smoker & NS \\
\hline Age & NS \\
\hline
\end{tabular}

$* N S=$ not significant. $†$ Significantly associated with the development of postoperative pulmonary dysfunction.

\section{Risk of Postoperative \\ Pulmonary Dysfunction}

Univariate and multivariate analyses were performed to look for any association between the development of postoperative pulmonary dysfunction and alveolar dead space, Injury Severity Score, pulmonary injury or comorbidity, smoking status, or age (Table II). We examined the correlation between postoperative pulmonary dysfunction and the predictor variables included in the multivariate models and found significant correlations with the measures of alveolar dead space at the three timeintervals ( $\mathrm{p}<0.001$ for all correlations) as well as with lung injury or comorbidity and the Injury Severity Score $(\mathrm{p}<$ $0.001)$. In the univariate analysis, one of the factors most strongly associated with the development of postoperative pulmonary dysfunction was the alveolar dead-space measurement at thirty minutes. No significant association was noted with the extent of reaming, the smoking status, or age. After controlling for important covariables, we found that the most important predictors of postoperative pulmonary dysfunction in the multivariate model were again the alveolar dead-space measurement at thirty minutes and the Injury Severity Score. The discriminant ability for this model correctly identified $80 \%$ of patients in whom postoperative pulmonary dysfunction would develop. There was no additional significant contribution from reaming, pulmonary injury or comorbidity, or a history of smoking. We re-examined the multivariate model after excluding patients who remained intubated for reasons possibly unrelated to pulmonary dysfunction. Similarly, the alveolar dead-space measurement at thirty minutes $(\mathrm{p}=0.005)$ and pulmonary injury or comorbidity ( $\mathrm{p}=0.01$ ) were the most important predictors of postoperative pulmonary dysfunction, despite the fact that the majority (six) of the eight excluded patients had had extensive reaming.

\section{Discussion}

Tntramedullary nailing has evolved 1 over the last forty years to become the preferred treatment for fractures of the femoral shaft, and it has been found to be associated with low morbidity rates and excellent clinical outcomes ${ }^{25,26}$. However, in a retrospective study of patients with femoral shaft fracture and thoracic injury who had undergone intramedullary nailing, Pape et al. found a higher prevalence of adult respiratory distress syndrome and a higher mortality rate in patients treated with reaming less than twenty-four hours after injury compared patients who had not had reaming ${ }^{3}$. Furthermore, Pape et al. compared the effects of intramedullary nailing with and without reaming in a prospective investigation of a small group of thirty-one patients with femoral shaft fracture but without thoracic injury ${ }^{20}$. They found a persistent and significant decrease in the oxygenation ratio in patients who underwent reaming, and they concluded that a nailing technique that does not involve reaming is indicated for patients with preexisting lung injury ${ }^{20}$. The results of this work led to other changes as well, including the use of small-diameter solid nails and alterations in the reamer $\operatorname{design}^{2-4}$.

Bosse et al. ${ }^{21}$ contradicted the work of Pape et al. In a retrospective study at two large metropolitan trauma centers, intramedullary nailing was compared with plate fixation for the treatment of femoral shaft fractures in patients with and without lung injury. There were no significant differences in the rates of adult respiratory distress syndrome or mortality between any of the groups, including patients with associated pulmonary injury. Bosse et al. concluded that the treatment of femoral shaft fractures with reaming and intramedullary nailing did not worsen the clinical or pulmonary outcome of patients with multiple injuries. Despite this positive report, there is a scarcity of data documenting pulmonary gas exchange and/ 
The JOURnAL of BOnE \& JOINT SURGERY • JBIS.ORG Volume 83-A · Number 8 - August 2001
Pulmonary Dysfunction in Patients with Femoral Shaft Fracture Treated With Intramedullary NaIling or early pulmonary outcomes in this patient population ${ }^{22}$.

The rationale for measuring alveolar dead space to demonstrate a functional consequence of pulmonary embolization has been well documented ${ }^{7-9,11,12,14}$. In an experimental study involving glass-bead embolization, alveolar dead space increased proportionate to the degree of pulmonary vascular obstruction in mechanically ventilated goats ${ }^{15}$. Other animal studies have suggested that an increase in the alveolar dead space occurs simultaneously with the onset of pulmonary vascular occlusion ${ }^{15,16}$. Measurement of alveolar dead space in humans has been examined as a method of screening for acute pulmonary embolism ${ }^{7,19}$. In 1990, Burki found measurement of physiologic dead-space fraction comparable, in terms of sensitivity and specificity, to radioisotope lung-scanning in the diagnosis of pulmonary embolism ${ }^{7}$.

Furthermore, experimental studies on the effect of pulmonary embolism have demonstrated an increase in alveolar dead space secondary to a reduction in perfusion of the affected lung ${ }^{16}$. Measurement of alveolar dead space is a sensitive screening test (sensitivity of $88 \%$ ) for acute pulmonary emboli in ambulatory patients ${ }^{12}$, and it has a high sensitivity and specificity for detection of pulmonary emboli in mechanically ventilated patients with intrinsic lung disease (asthma, chronic obstructive pulmonary disease, and so on $)^{9,11}$. Thus, there is ample evidence suggesting that pulmonary gas exchangespecifically, alveolar dead space-should change if a large embolic burden is routinely induced by reaming and/or nailing of a fractured femoral shaft.

Recently, Weresh et al. sought to determine if nailing of femoral shaft fractures produces immediate worsening of pulmonary gas exchange $e^{22}$. They found no significant change in the alveolar dead space during or shortly following nailing, and they concluded that intramedullary nailing of femoral shaft fractures does not routinely produce a large enough burden of pulmonary embolization to significantly change pulmonary gas exchange (specifically, alveolar dead space).

We prospectively studied a consecutive series to determine intermediate-term pulmonary and clinical outcomes in patients with a femoral shaft fracture treated with intramedullary nailing. We believe that we are the first to define an important relationship between alveolar dead space and specific pulmonary clinical outcomes in such patients. Our definition of postoperative pulmonary dysfunction was chosen to identify patients who would clearly benefit from reevaluation by a physician or from intensive-care monitoring postoperatively. Thus, the fifty-four patients in our series who had no signs of postoperative pulmonary dysfunction could have been safely managed on a nonmonitored orthopaedic ward. This study's strength lies in our ability to evaluate several important confounding variables that affect pulmonary function, such as pulmonary contusion, hemothorax, pneumothorax, chronic obstructive pulmonary disease, asthma, and smoking. Unlike in previous studies, the number of patients with postoperative pulmonary dysfunction in our study provided adequate statistical power for us to evaluate several important predictors of this outcome. The most controversial of these, exten- sive reaming of the medullary canal, was not associated with postoperative pulmonary dysfunction. Other, more important predictors of postoperative pulmonary dysfunctionthat is, alveolar dead space, Injury Severity Score, and concomitant pulmonary injury-were identified. These data should be validated in a prospective fashion. Furthermore, we examined the impact of excluding from the analysis eight patients who had remained intubated for reasons not attributed to known pulmonary dysfunction. The results of our predictive model were robust; again, alveolar dead space and lung injury were the major predictors of postoperative pulmonary dysfunction.

Our study had several weaknesses. Although the sample size allowed evaluation of several important predictor variables, a larger sample might have increased our ability to identify other factors contributing to postoperative pulmonary dysfunction. We could not control for the selection of patients for either minimal or extensive reaming. Other, unmeasured factors may play a role in this decision and thus in patient outcome. Nevertheless, we identified the association between the objective score for overall patient injury and the outcome, which may partially address this concern. Validation of our results in a separate study would be useful.

Alveolar dead-space measurements did not increase significantly over time in patients who underwent extensive femoral reaming. We interpreted these findings to indicate that extensive reaming of the femoral canal does not pose an increased threat to pulmonary function in patients with a femoral fracture. Furthermore, the addition of reaming did not increase the risk of postoperative pulmonary dysfunction according to univariate or multivariate analysis. This was true regardless of potential confounding variables, including associated pulmonary injury, preexisting pulmonary disease, and a positive smoking history. These results suggest that the concern that the process of reaming the femoral canal will worsen pulmonary function in a patient with multiple injuries may be unfounded.

Additionally, we found that an increased alveolar dead space prior to nailing is predictive of postoperative pulmonary dysfunction. Even more important is the alveolar dead-space measurement obtained thirty minutes after opening of the femoral canal. This single measurement, if increased by $>20 \%$, is highly predictive of postoperative pulmonary dysfunction.

Finally, our study results suggest that patients who undergo minimal reaming of the femoral canal have greater alveolar dead space and greater risk for the development of postoperative pulmonary dysfunction than do those who undergo extensive reaming. We speculate that a selection bias may have occurred in our study. At our institution, femoral nailing with minimal reaming is reserved for severely injured or clinically unstable patients. It is likely that the condition of these patients was considered to be more unstable than that of the patients who underwent extensive reaming. However, on analysis of such covariables as the Injury Severity Score, the degree of pulmonary injury, and pulmonary comorbidities, 
The Journal of Bone \& JOINT SURGERY · JBJS.ORG Volume 83-A · Number $8 \cdot$ August 2001
Pulmonary Dysfunction in Patients with Femoral Shaft Fracture Treated With Intramedullary Nailing no difference was noted between the group treated with reaming and that treated without reaming. This finding suggests that other variables, unknown and uncontrolled, likely influenced the attending physician's decisions regarding reaming in the treatment of the femoral shaft fractures in these patients with multiple injuries.

Brent L. Norris, MD

Joseph N. Rudd Jr., BSN, PhD

Department of Orthopaedic Surgery, University of Tennessee College of Medicine, Chattanooga Unit, 975 East Third Street, Box 287, Chattanooga, TN 37403. E-mail address for B.L. Norris: norrisbl@erlanger.org

W. Christopher Patton, MD
Alabama Orthopaedic Clinic, 271 Azalea Road, Mobile, AL 36609. E-mail address:wcpattonmd@aol.com

Colleen M. Schmitt, MD, MHS

Department of Internal Medicine, University of Tennessee College of Medicine, Chattanooga Unit, 975 East Third Street, Suite 505, Chattanooga, TN 37403

Jeffrey A. Kline, MD

Department of Emergency Medicine, Carolinas Medical Center, MEB 304, 1000 Blythe Boulevard, Charlotte, NC 28232-2861

No benefits in any form have been received or will be received from a commercial party related directly or indirectly to the subject of this article. Funds were received in total or partial support of the research or clinical study presented in this article. The funding sources were a Charlotte-Mecklenburg Hospital Authority Grant and the Chattanooga Orthopaedic Educational Research Foundation.

\section{References}

1. Boulanger BR, Stephen D, Brenneman FD. Thoracic trauma and early intramedullary nailing of femur fractures: are we doing harm? J Trauma. 1997;43:24-8

2. Pape HC, Regel G, Dwenger A, Sturm JA, Tscherne H. Influence of thoracic trauma and primary femoral intramedullary nailing on the incidence of ARDS in multiple trauma patients. Injury. 1993;24 Suppl 3:S82-103.

3. Pape HC, Regel G, Dwenger A, Krumm K, Schweitzer G, Kretteck C, Sturm JA, Tscherne H. Influences of different methods of intramedullary femoral nailing on lung function in patients with multiple trauma. $J$ Trauma. 1993;35:709-16.

4. Pape HC, Auf'm'Kolk M, Paffrath T, Regel G, Sturm JA, Tscherne H. Primary intramedullary femur fixation in multiple trauma patients with associated lung contusion-a cause of posttraumatic ARDS? J Trauma. 1993;34:540-7.

5. Talucci RC, Manning J, Lampard S, Bach A, Carrico JC. Early intramedullary nailing of femoral shaft fractures: a cause of fat embolism syndrome. Am J Surg. 1983;146:107-11.

6. Pell AC, Christie J, Keating JF, Sutherland GR. The detection of fat embolism by transesophageal echocardiography during reamed intramedullary nailing. A study of 24 patients with femoral and tibial fractures. J Bone Joint Surg Br. 1993;75:921-5.

7. Burki NK. Diagnosing pulmonary embolism. Chest. 1990;98:777-8.

8. Carroll GC. Capnographic trend curve monitoring can detect 1-ml pulmonary emboli in humans. J Clin Monit. 1992;8:101-6.

9. Chopin C, Fesard P, Mangalaboyi J, Lestavel P, Chambrin MC, Fourrier F, Rime A. Use of capnography in diagnosis of pulmonary embolism during acute respiratory failure of chronic obstructive pulmonary disease. Crit Care Med. 1990;18:353-7.

10. Elliott CG. Pulmonary physiology during pulmonary embolism. Chest. 1992;101(4 Suppl):163S-71S.

11. Hatle L, Rokseth $\mathbf{R}$. The arterial to end-expiratory carbon dioxide tension gradient in acute pulmonary embolism and other cardiopulmonary diseases. Chest. 1974;66:352-7.

12. Kline JA, Meek S, Boudrow D, Warner D, Colucciello S. Use of the alveola dead space fraction $(\mathrm{Vd} / \mathrm{Vt})$ and plasma D-dimers to exclude acute pulmonary embolism in ambulatory patients. Acad Emerg Med. 1997;4:856-63.

13. Santolicandro A, Prediletto R, Fornai E, Formich B, Begliomini E, GianellaNeto A, Giuntini C. Mechanisms of hypoxemia and hypocapnia in pulmonary embolism. Am J Respir Crit Care Med. 1995;152:336-47.

14. Sasahara AA, Cannilla JE, Morse RL, Sidd JJ, Tremblay GM. Clinical and physiologic studies in pulmonary thromboembolism. Am J Cardiol. 1967;20:10-20.

15. Schreiner MS, Leksell LG, Gobran SR, Hoffman EA, Scherer PW, Neufeld GR. Microemboli reduce phase III slopes of $\mathrm{CO} 2$ and invert phase III slopes of infused SF6. Respir Physiol. 1993;91:137-54.

16. Severinghaus JW, Stupfel M. Alveolar dead space as an index of distribution of blood flow in pulmonary capillaries. J Appl Physiol. 1957;10:335-48.

17. Wilson RF, Tyburski JG, Kubinec SM, Warsow KM, Larky HC, Wilson SR, Schermerhorn T. Intraoperative end-tidal carbon dioxide levels and derived calculations correlated with outcome in trauma patients. $J$ Trauma. 1996;41:606-11.

18. Duwelius PJ, Huckfeldt R, Mullins RJ, Shiota T, Woll TS, Lindsey KH, Wheeler $\mathbf{D}$. The effects of femoral intramedullary reaming on pulmonary function in a sheep lung model. J Bone Joint Surg Am. 1997;79:194-202.

19. Heim D, Regazzoni P, Tsakiris DA, Aebi T, Schlegel U, Marbet GA, Perren SM. Intramedullary nailing and pulmonary embolism: does unreamed nailing prevent embolism? An in vivo study in rabbits. J Trauma. 1995;38:899-906.

20. Pape HC, Dwenger A, Grotz M, Kaever V, Negatsch R, Kleeman W, Regel G, Sturm JA, Tscherne H. Does the reamer type influence the degree of lung dysfunction after femoral nailing following severe trauma? An animal study. J Orthop Trauma. 1994;8:300-9.

21. Bosse MJ, MacKenzie EJ, Riemer BL, Brumback RJ, McCarthy ML, Burgess AR, Gens DR, Yasui Y. Adult respiratory distress syndrome, pneumonia, and mortality following thoracic injury and a femoral fracture treated either with in tramedullary nailing with reaming or with a plate. A comparative study. JBone Joint Surg Am. 1997;79:799-809.

22. Weresh MJ, Stover MD, Bosse MJ, Jeray K, Kellam JF, Sims SH, Kline JA Pulmonary gas exchange during intramedullary fixation of femoral shaft fractures. J Trauma. 1999;46:863-8.

23. Domsky M, Wilson RF, Heins J. Intraoperative end-tidal carbon dioxide values and derived calculations correlated with outcome: prognosis and capnography. Crit Care Med. 1995;9:1497-503.

24. Enghoff H. Volumen ineffeicax. Bemerkungen zur Frage des schädlichen Raumes. Upsala: Läkaref Förhandl. 1938;44:191-218.

25. Winquist RA, Hansen ST Jr, Clawson DK. Closed intramedullary nailing of femoral fractures. A report of five hundred and twenty cases. $J$ Bone Joint Surg Am. 1984;66:529-39.

26. Winquist RA, Hansen ST Jr. Comminuted fractures of the femoral shaft treated by intramedullary nailing. Orthop Clin North Am. 1980;11:633-48. 